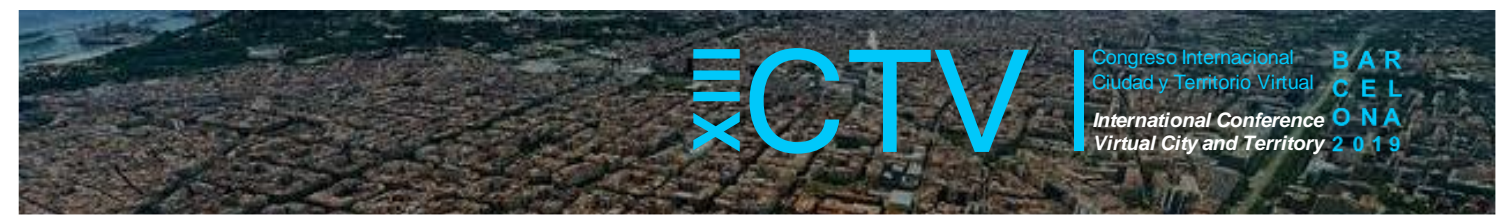

\title{
ESTRATEGIAS DE PARTICIPACIÓN CIUDADANA Y NUEVAS TECNOLOGÍAS. APROXIMACIÓN ACTUAL A LOS URBAN CENTERS Y CENTROS DE INNOVACIÓN URBANA
}

\author{
Berigüete Alcántara, Fanny Esther ${ }^{1 *}$; Rodríguez Cantalapiedra, Inmaculada ${ }^{2}$ y Mayorga \\ Cárdenas, Miguel Yury ${ }^{3}$
}

Remisión inicial: 2019-06-14; Remisión definitiva: 2019-10-20; Publicación: 2019-12-21

Citación: Berigüete Alcántara, F. et al. (2019). Estrategias de participación ciudadana y nuevas tecnologías. Aproximación actual a los urban centers y centros de innovación urbana. En XIII CTV 2019 Proceedings: XIII International Conference on Virtual City and Territory: "Challenges and paradigms of the contemporary city": UPC, Barcelona, October 2-4, 2019. Barcelona: CPSV, 2019, p. 8553. E-ISSN 2604-6512. DOI http://dx.doi.org/10.5821/ctv.8553

\begin{abstract}
Resumen
En los últimos años se ha ratificado la necesidad de implementar una nueva democracia participativa en los asuntos urbanos, donde los ciudadanos gestionen, informen, reflexionen y planteen soluciones ante los problemas actuales y futuros de las ciudades. Los Urban Centers o centros de innovación urbana están surgiendo como herramientas en pro de la construcción de políticas urbanas colaborativas mediante el fortalecimiento del rol activo de los ciudadanos con intereses comunes, con una visión local y global. El objetivo principal a desarrollar en la ponencia es la conceptualización y aproximación en torno a los urban center y su estado actual, tomando como referencia el caso de Bolonia, Barcelona y Boston, además de establecer la relación actual entre lo presencial y lo digital en la participación ciudadana.
\end{abstract}

Se ha recopilado información documental sobre los $\mathrm{UC},{ }^{2}$ haciendo énfasis en el papel que tienen éstos en la participación activa y directa de los ciudadanos, que nos permite entender el fenómeno y sus variantes y las posibilidades que pueden resultar de su aplicación en las esferas de la participación ciudadana. Además de ello, para poder profundizar más en la implementación de esta participación, se plantea una metodología que permita determinar las estrategias necesarias para ello, con el apoyo de los colectivos implicados en este proceso y el uso de las nuevas plataformas y medios digitales. Dicha metodología se divide en tres fases, que van desde el diagnóstico actual, hasta la generación colectiva de propuestas; dichos datos serán obtenidos y procesados con plataformas de encuestas y participación, además de mesas de trabajo presenciales.

Al analizar los resultados, nos podemos percatar de la poca divulgación que ha tenido este tipo de mecanismo de participación ciudadana, alcanzando su mayor difusión y puesta en práctica en Italia, siendo el UC de Bolonia el punto de referencia. Un caso interesante es el de Boston, el cual está en la transición de un centro a una red de centros, lo que permite el intercambio de experiencias, plantear problemas y soluciones comunes y satisfacer las necesidades específicas y generales de la ciudad y sus ciudadanos. En el caso de Barcelona, se está implementando un UC donde se fomenta la innovación urbana en los ámbitos social, urbanístico, económico y tecnológico. Estas buenas prácticas, aunque necesitan expandirse y renovarse, pueden servir como plataforma para la creación de una red de forma territorialmente descentralizada de puntos de referencia de participación ciudadana y de prácticas colectivas urbanas. A partir de esto, es importante seguir tres líneas: transformar los ya existentes, incluyendo a los ciudadanos no sólo como espectadores, sino como agentes activos en la conformación de la ciudad, a través de talleres, seminarios, debates, plataformas digitales, documentación, centros de exposición y mesas de trabajos, de la mano de las nuevas tecnologías de cara al usuario-ciudadano; crear una red más amplia de UC, interconectadas unas con otras, para intercambiar experiencias y buenas prácticas, y crear herramientas o apoyarnos en las ya existentes, para una participación en tiempo real, más transparente y accesible. Esto supone mayor cobertura e información de los procesos actuales de participación.

\footnotetext{
${ }^{1}$ Departamento de Física, Escuela Superior de Edificación de Barcelona-EPSEB, Universitat Polítècnica de CatalunyaUPC, Barcelona, España, https://orcid.org/0000-0003-4887-2062; ${ }^{2}$ Departamento de Física, Escuela Superior de Edificación de Barcelona-EPSEB, Universitat Polítècnica de Catalunya-UPC, Barcelona, España, http://orcid.org/00000002-0070-8979; ${ }^{3}$ Departamento de Ingeniería Civil y Ambiental, Universidad Politécnica de Cataluña, Barcelona, España, http://orcid.org/0000-0002-3389-3541. * Correo de contacto: fanny.esther.beriguete@upc.edu ${ }^{2}$ Urban Centers
} 


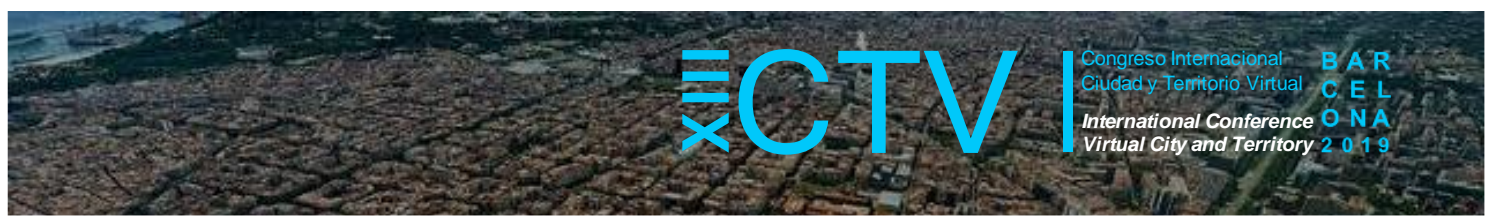

\section{Abstract}

In recent years the need to implement a new participatory democracy in urban affairs has been ratified, where citizens manage, inform, reflect and propose solutions to the current and future problems of cities. Urban Centers or urban innovation centers are emerging as tools for the construction of collaborative urban policies by strengthening the active role of citizens with common interests, with a local and global vision. The main objective to develop in the paper is the conceptualization and approach around the urban center and its current state, taking as a reference the case of Bologna, Barcelona and Boston, in addition to establishing the current relationship between the face-to-face and the digital in the citizen participation.

Documentary information on the UC has been collected emphasizing their role in the active and direct participation of citizens, which allows us to understand the phenomenon and its variants and the possibilities that may result from its application in the areas of participation Citizen In addition to this, to be able to deepen more in the implementation of this participation, a methodology is proposed that allows determining the necessary strategies for this, with the support of the groups involved in this process and the use of the new platforms and digital media. This methodology is divided into three phases, ranging from the current diagnosis, to the collective generation of proposals; these data will be obtained and processed with survey and participation platforms, as well as face-to-face work tables.

When analyzing the results, we can notice the little dissemination that this type of citizen participation mechanism has had, reaching its greater dissemination and implementation in Italy, the UC of Bologna being the reference point. An interesting case is that of Boston, which is in the transition from a center to a network of centers, allowing the exchange of experiences, raising common problems and solutions and meeting the specific and general needs of the city and its citizens. In the case of Barcelona, a UC is being implemented where urban innovation is promoted in the social, urban, economic and technological fields. These good practices, although they need to be expanded and renewed, can serve as a platform for the creation of a territorially decentralized network of citizen participation and urban collective practices benchmarks. From this, it is important to follow three lines: transform existing ones, including citizens not only as spectators, but as active agents in the shaping of the city, through workshops, seminars, debates, digital platforms, documentation, exhibition centers and work tables, hand in hand with new technologies for the user-citizen; create a wider network of UC, interconnected with each other, to exchange experiences and good practices, and create tools or rely on existing ones, for a real-time, more transparent and accessible participation. This means greater coverage and information on the current participation processes.

Palabras clave: urban centers; participación ciudadana; casas de la ciudad; nuevas tecnologías

Key words: urban centers; citizen participation; city home; news technologies

\section{Introducción}

La participación ciudadana se hace cada vez más necesaria para afrontar las problemáticas actuales, cuya finalidad son la inclusión de estos en la definición de proyectos y programas urbanos, la promoción de iniciativas y voluntariados que estimulen el involucramiento activo de todos los actores de la ciudad y afianzar y asegurar una sociedad más inclusiva, tal como señala Fernández González M. (2015) se "hace urgente una participación activa y amplia de las diferentes disciplinas que deberían sentirse llamadas a pensar la ciudad". Para ello Borja y Castells (1997) proponen siete principios para que la participación sea más democrática: "1) Derecho a la participación en la vida internacional; 2)Derecho a la innovación política en las formas de gestión de servicios; 3)Derecho a asumir competencias tradicionalmente propias de la administración central o del sector privado; 4) Los gobiernos locales deben garantizar a los ciudadanos la igualdad política y jurídica, sin importar su origen; 5) Igualdad de género; 6)Los gobiernos locales promoverán la vida colectiva y el asociacionismo y 7)Finalmente la ciudad, su gobierno y su sociedad civil, asumirán su función de centralidad en los ámbitos regional y nacional, garantizando el uso de la cuidad a sus ciudadanos". (p.302). Los urban centers surgen como espacio que promueven el conocimiento y la participación colectiva, es por ello, que este proyecto se enfoca en ellos, como medios para lograr una participación más integral. 


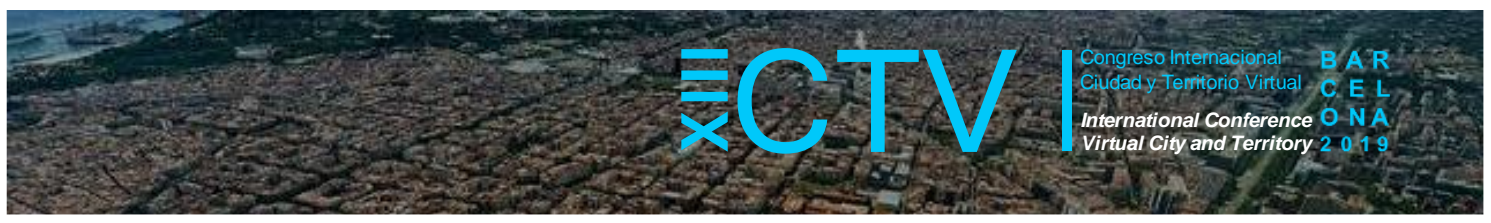

La tecnología en este ámbito, también sirve de canal para la participación, tal como señala Sierra Caballero y Del Valle (2007) existen muchas experiencias internacionales donde se potencia la participación ciudadana a través de internet, pasando de consumidor a agente activo en los procesos de creación y desarrollo económico y cultural. El principio "hazlo tú mismo" reivindica las formas de producción participativa, además, de crear las bases para la acción ciudadana en los aspectos urbanos. De manera general, se puede decir que esta transformación digital nos facilita diversos canales, medios y recursos para lograr una cocreación del espacio y un empoderamiento colectivo.

\section{Metodología}

En un primer momento, se establecerán los conceptos y divergencias de este término, se analizan las diferentes experiencias que existen en este ámbito y su extrapolación entre diferentes ciudades europeas y americanas; esto nos permite entender lo que conlleva este concepto y su estado actual. Seguidamente analizaremos en profundidad los aportes e inconvenientes que se pueden obtener con esta herramienta física de participación. Por último, se establecerá una relación entre los centros urbanos como estrategia de participación y las nuevas herramientas y aplicaciones de participación ciudadana (Fig. 1).

Figura 1. Análisis de la información

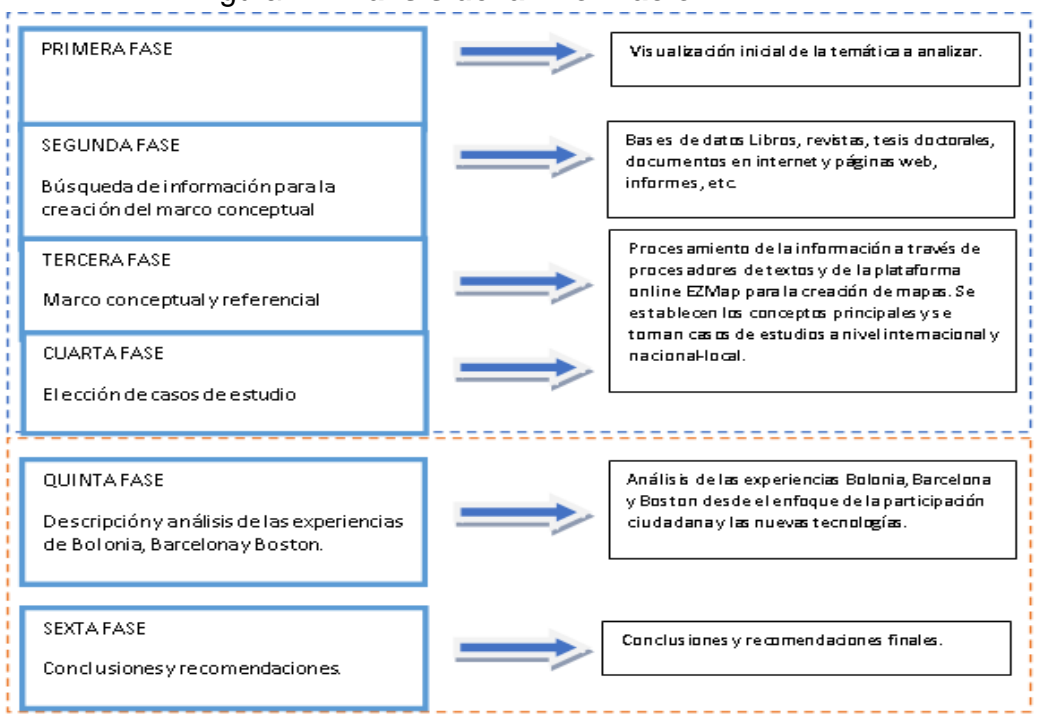

Fuente: Elaboración propia

\subsection{Metodología del proceso de participación}

Se propone una estructura de tres fases:

- Fase de diagnosis y diseño

Esta fase se inicia con una valoración de la participación ciudadana en los UC, a través de una encuesta online con preguntas claves que indagan sobre si los ciudadanos conocen esta herramienta de participación y de ser así cómo lo hacen. Para ello se elabora un plan de comunicación para involucrar a la mayor cantidad de agentes y obtener resultados más fidedignos. Además de ello, se involucra a expertos en el tema de la participación. 


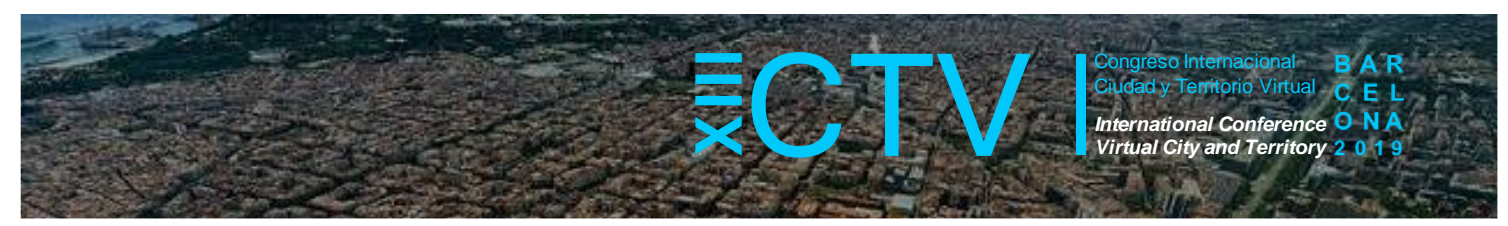

\section{- Fase de Ejecución}

Después del diseño de la encuesta se elige la plataforma ${ }^{3}$ que la albergará. Esta permitirá comparar la realidad actual con la percepción de los ciudadanos y nos dará un punto de partida para implementar las estrategias necesarias para aumentar dicha participación.

Una vez obtenido estos resultados se realiza un debate online ${ }^{4}$ donde los diferentes actores expresan sus percepciones en cuanto al tema en cuestión y aportan ideas para aumentar y viabilizar dicha participación. Esta etapa es interesante, ya que va en consonancia con la creación colectiva que se quiere lograr en el UC. Además de este debate online, se pueden realizar talleres de generación de ideas ${ }^{5}$ en los urban centers.

Finalizado el debate online se procede a analizar las propuestas, seleccionando aquellas que sean más viables, para lo cual se puede realizar una reunión colectiva para consensuar las mejores propuestas.

\section{- Fase Final}

Se redacta un informe con los principales resultados y recomendaciones obtenidas del proceso, no sólo basándonos en las propuestas recibidas, sino también de la efectividad de la metodología utilizada. Para el seguimiento de estas propuestas y su ejecución se recomienda la creación de una plataforma ${ }^{3}$ de participación que nos permita la comunicación a través de newsletter con los participantes.

\section{Concepto}

En esta investigación se hace referencia al termino UC como un laboratorio de participación ciudadana ubicado en centros neurálgicos de la ciudad. "Urban Center es un concepto utilizado para describir cualquier institución cuya misión principal es informar y comprometer a los ciudadanos en la planificación urbana y las políticas públicas"(Observatorio de investigación del centro urbano, s. f.).

Este concepto tiene su origen en los EE. UU. “...como un lugar físicamente central designado institucionalmente para informar, comunicar y discutir proyectos de transformación urbana, los Urban Centers tienen como objetivo crear un foro de debate para administradores, profesionales, empresarios, grupos sociales, comités de ciudadanos e individuos que desean participar activamente. Contribuir a dar forma al futuro de la ciudad". (Observatorio de investigación del centro urbano, s. f.).

Estos espacios centrales son puntos de confluencias de los diversos actores que intervienen en la ciudad, aportando cada uno desde su conocimiento y realidad soluciones a las problemáticas que afectan a la ciudad. Este tipo de participación es presencial, por lo que permite una relación directa entre todas las personas que forman parte del proceso. La creación de UC está supeditada a las variantes culturales de administración de las ciudades que producen diversas formas de interpretación, de acuerdo a dos categorías de derechos: derecho civil (que es el

\footnotetext{
3 Se analiza la idoneidad de las plataformas Survey Monkey https://es.surveymonkey.com/ y Survio https://www.survio.com/ para la realización de la encuesta, ambas permiten el acceso a la plataforma a través de smartphone, tablets y computador.

${ }^{4}$ Plataforma Kuorum https://kuorum.org/en/ o Decidim https://decidim.org/

${ }^{5}$ Sesiones presenciales donde los participantes a través de dinámicas grupales generan ideas en torno a una temática para explorar la máxima cantidad de aspectos primordiales sobre el tema.
} 


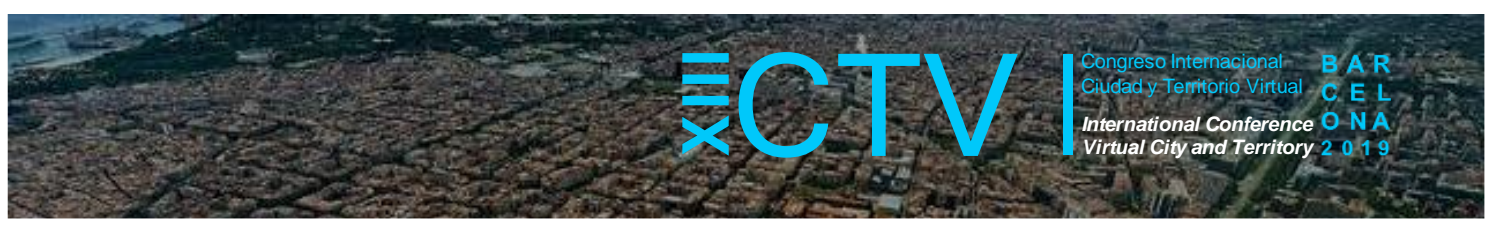

derecho más utilizado mundialmente) y el derecho común. En los países de "Derecho civil", las iniciativas son gestionadas por el gobierno local, ya sea en solitario o en colaboración con instituciones internacionales y/o privadas.

En los países de "derecho común", el contexto legal y cultural fomenta la creación de UC gestionados por agentes neutrales, que no pertenecen a la administración local (instituciones académicas, organizaciones sin fines de lucro, asociaciones comunitarias, empresas, fundaciones)(Observatorio de investigación del centro urbano, s. f.). Es interesante ver cómo se puede manifestar este fenómeno de la mano de uno u otro actor, dependiendo del sistema legal que predomine en éste. En la Figura 2 se puede ver cuáles países pertenecen al derecho común (Common law) y al derecho civil (Derecho continental).

Figura 2. Mapa de distribución de los modelos de derecho

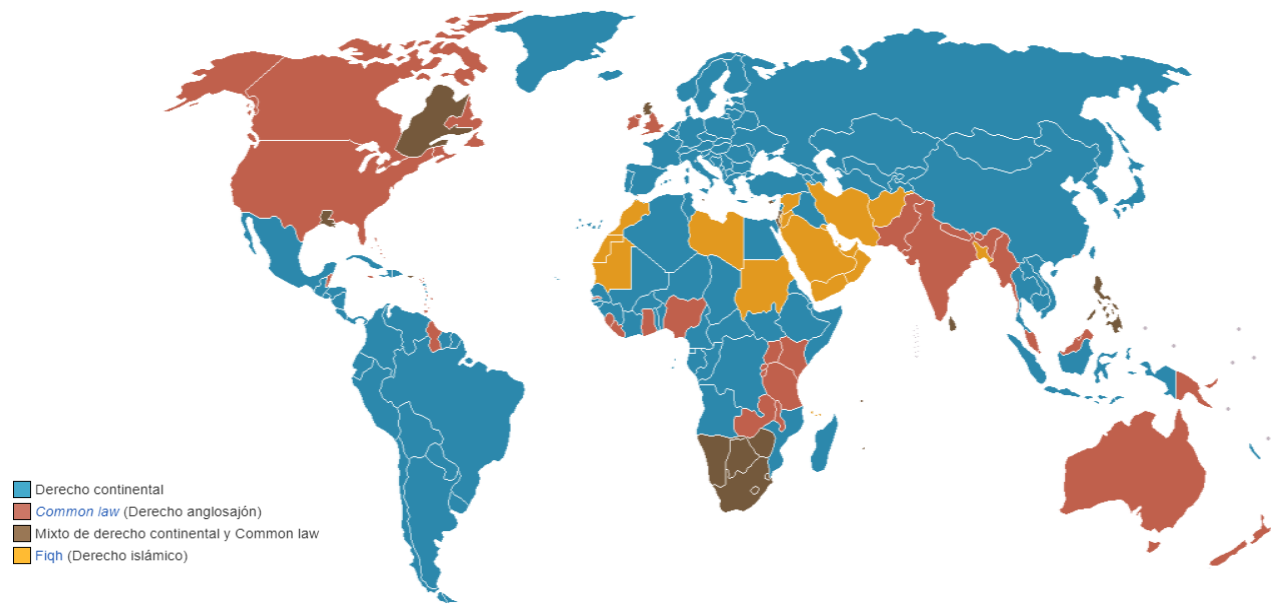

Fuente: (Wikipedia Commons, 2005), disponible en: https://es.wikipedia.org/wiki/Derecho_continental

La superación de la dicotomía público / privado tradicional ha promovido una audiencia más amplia de partes interesadas en el proceso de toma de decisiones, en el que la dialéctica entre entidades establecidas (autoridades locales, grupos empresariales e inversionistas financieros) y "actores emergentes" (sociedades especializadas, sin fines de lucro, organizaciones, grupos de interés común, etc.) pueden consolidarse en un Centro Urbano: "casas de cristal" para escenarios de desarrollo de ciudades compartidas. El interés en el fenómeno de la UC está vinculado a la evolución que estas estructuras pueden representar para las autoridades gubernamentales locales. Pueden convertirse en una oportunidad para experimentar con nuevas formas de democracia participativa y deliberativa, no solo limitadas a los destinatarios pasivos de la comunicación, sino que apuntan a la construcción compartida de directrices para la política urbana (Observatorio de investigación del centro urbano, s. f.).

\section{Estado Actual}

De acuerdo al Osservatorio di ricerca sugli urban center ${ }^{6}$ existen 44 UC distribuidos en América del Norte y Europa (Fig. 3), de los cuales 3 se encuentran inactivos en la actualidad (Observatorio de investigación del centro urbano, s. f.).

\footnotetext{
${ }^{6}$ Es un centro de investigación permanente en centros urbanos cuya misión principal profundizar el estudio sobre los Centros Urbanos como lugares privilegiados de democracia participativa y espacios neutrales de deliberación compartida para la construcción de políticas urbanas.
} 
Figura 3. Distribución de los UC

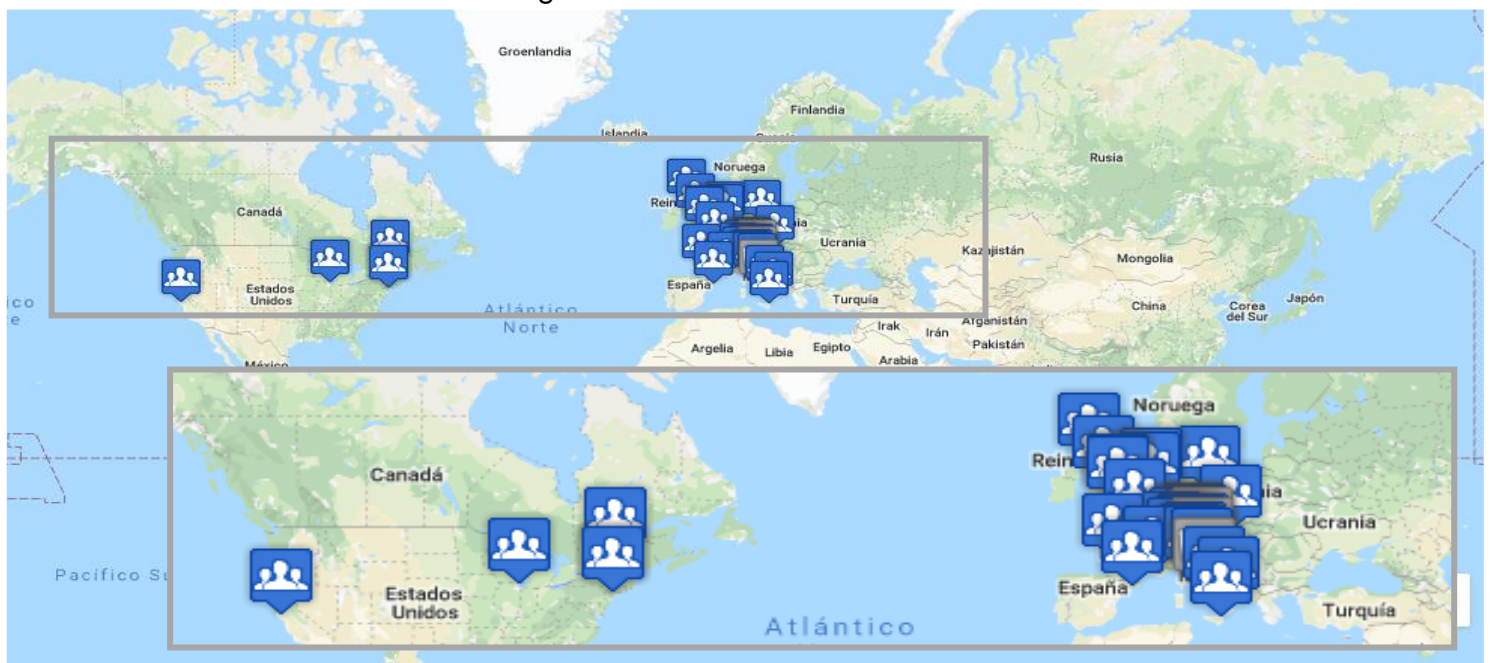

Fuente: Elaboración propia mediante EZMap

Figura 4. Distribución de los UC en América del Norte

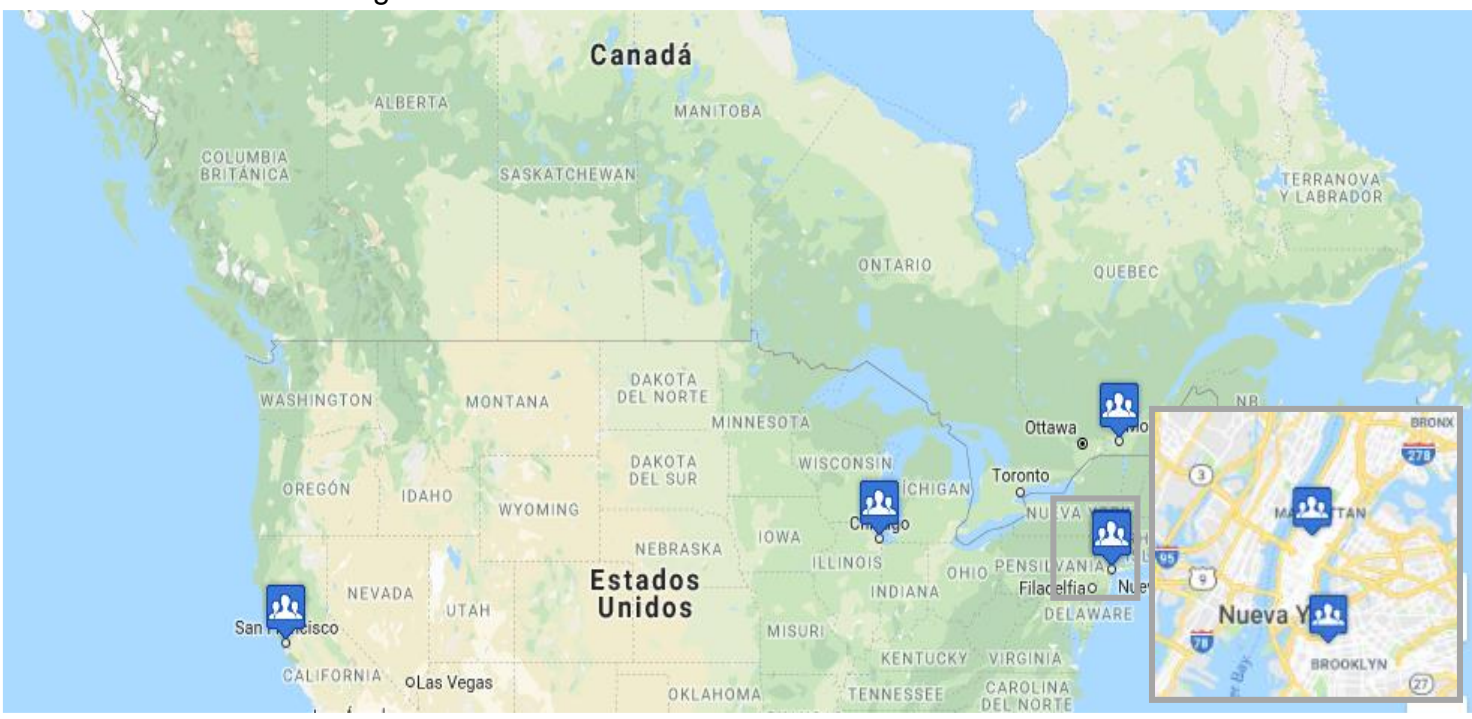

Fuente: Elaboración propia mediante EZMap

Tabla 1. Nombre e información de contacto de los UC en América del Norte ${ }^{7}$

\begin{tabular}{ll}
\hline Canadá & \\
\hline CCA - Centre Canadien d'Architecture - Montrèal & sitio web: www.cca.qc.ca \\
\hline EE.UU & \\
\hline The Municipal Art Society of New York & sitio web: www.mas.org \\
\hline Pratt Center for Community Development - New York & sitio web: www.prattcenter.net \\
\hline San Francisco Planning and Urban Research Association (SPUR) & sitio web: www.spur.org \\
\hline Chicago Architecture Foundation (CAF) & sitio web: www.architecture.org \\
\hline
\end{tabular}

Fuente: Elaboración propia a partir de datos del Observatorio de investigación de Centros Urbanos

\footnotetext{
${ }^{7}$ En la Figura 4 y la Tabla 1 no se muestra el UC de Boston, ya que estas gráficas fueron realizadas con datos del
} Observatorio de investigación de centros urbanos y este no está adscrito al mismo. 
Figura 5. Distribución de los UC en Francia y España

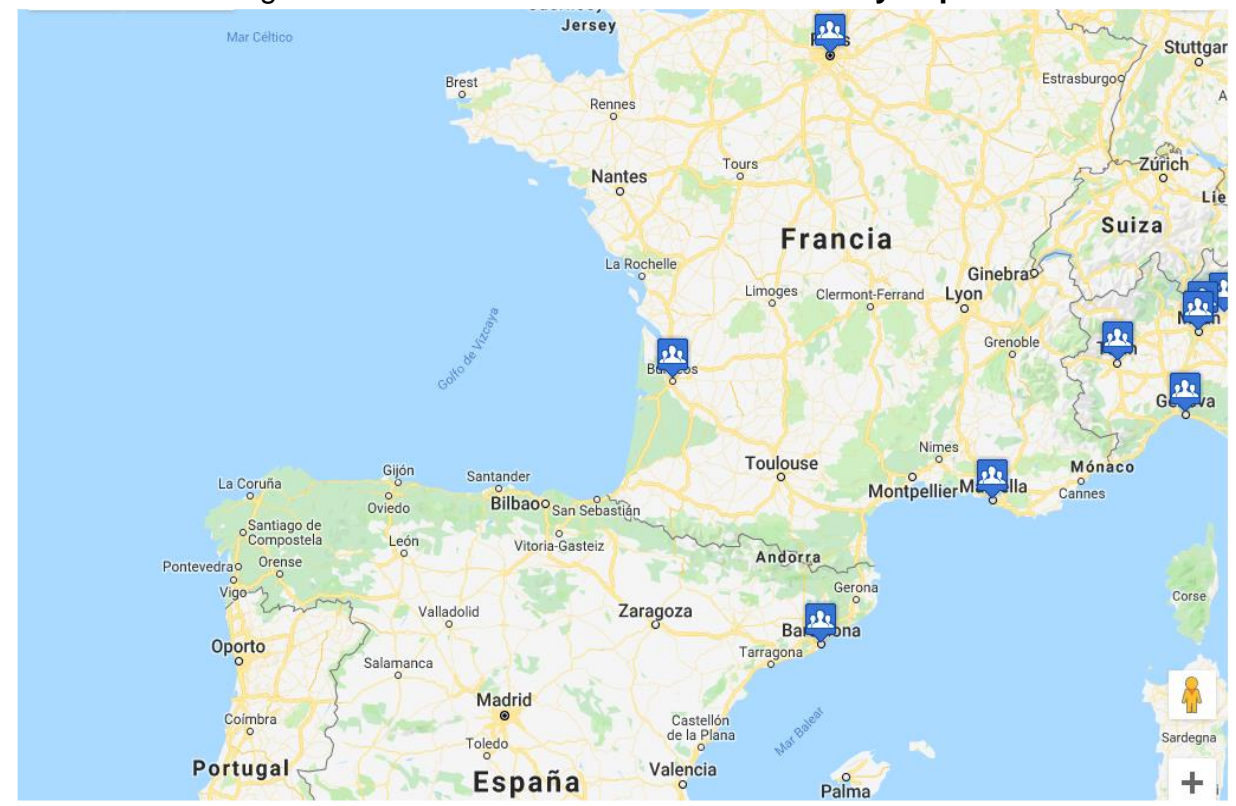

Fuente: Elaboración propia mediante EZMap

Tabla 2. Nombre e información de contacto de los UC en Francia y España

\section{Francia}

\begin{tabular}{ll}
\hline Pavillon de l'Arsenal - Paris & sitio web: www.pavillon-arsenal.com \\
\hline Arc-en-Reve - Bordeaux & sitio web: www.arcenreve.com \\
\hline Bureau de Comptences et Desirs - Marseille & sitio web: www.bureaudescompetences.org \\
\hline España & \\
\hline Centre de Cultura Contemporània de Barcelona & sitio web: www.cccb.org \\
\hline
\end{tabular}

Fuente: Elaboración propia a partir de datos del Observatorio de investigación de Centros Urbanos

Figura 6. Distribución de los UC en Reino Unido, Alemania, Países Bajos y Austria

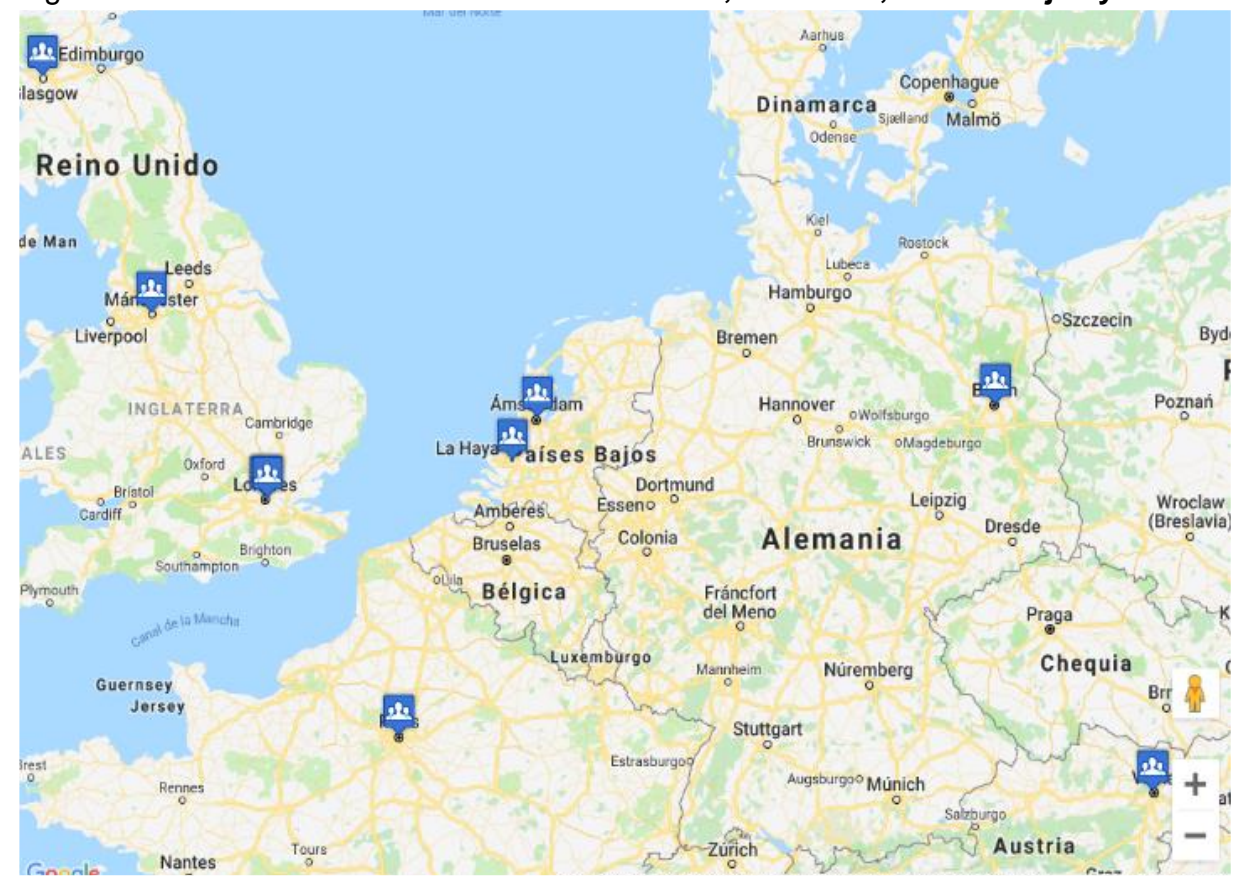

Fuente: Elaboración propia mediante EZMap 


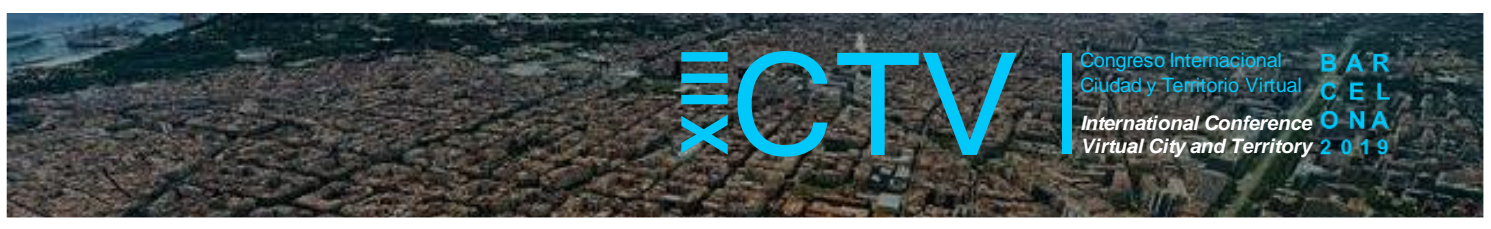

Tabla 3. Nombre e información de contacto de los UC en Reino Unido, Alemania, Países Bajos y Austria

\begin{tabular}{ll}
\hline Reino Unido & \\
\hline New London Architecture - London & sitio web: www.newlondonarchitecture.org \\
\hline London Openhouse - London & sitio web: www.londonopenhouse.org \\
\hline Design for London - London & sitio web: www.designforlondon.gov.uk \\
\hline The Cube - Manchester & sitio web: www.cube.org.uk \\
\hline The Lighthouse - Glasgow & sitio web: www.thelighthouse.co.uk \\
\hline Alemania & \\
\hline Deutsches Architektur Zentrum - Berlin & sitio web: www.daz.de \\
\hline Paises Bajos & \\
\hline NAl - Netherland Architecture Institute - Rotterdam & sitio web: www.nai.nl \\
\hline Architectuurcentrum Amsterdam (ARCAM) & sitio web: www.arcam.nl \\
\hline Austria & \\
\hline Architekturzentrum Wien (AZW) & sitio web: www.azw.at \\
\hline
\end{tabular}

Fuente: Elaboración propia a partir de datos del Observatorio de investigación de Centros Urbanos

Como se puede observar en la Figura 7 y en la Tabla 4, Italia es uno de los países que está a la cabeza en la gestión y estudio de los UC como escenarios de participación ciudadana en ciudades como Bolonia, Cosenza, Nápoles, Ferrara, Parma, Roma, Génova, Bérgamo, Brescia, Milán, Monza, Turín, Catania, Pontedera, Siena, Florencia, Trento, Rovereto, Perugia y Vicenza.

Figura 7. Distribución de los UC en Italia

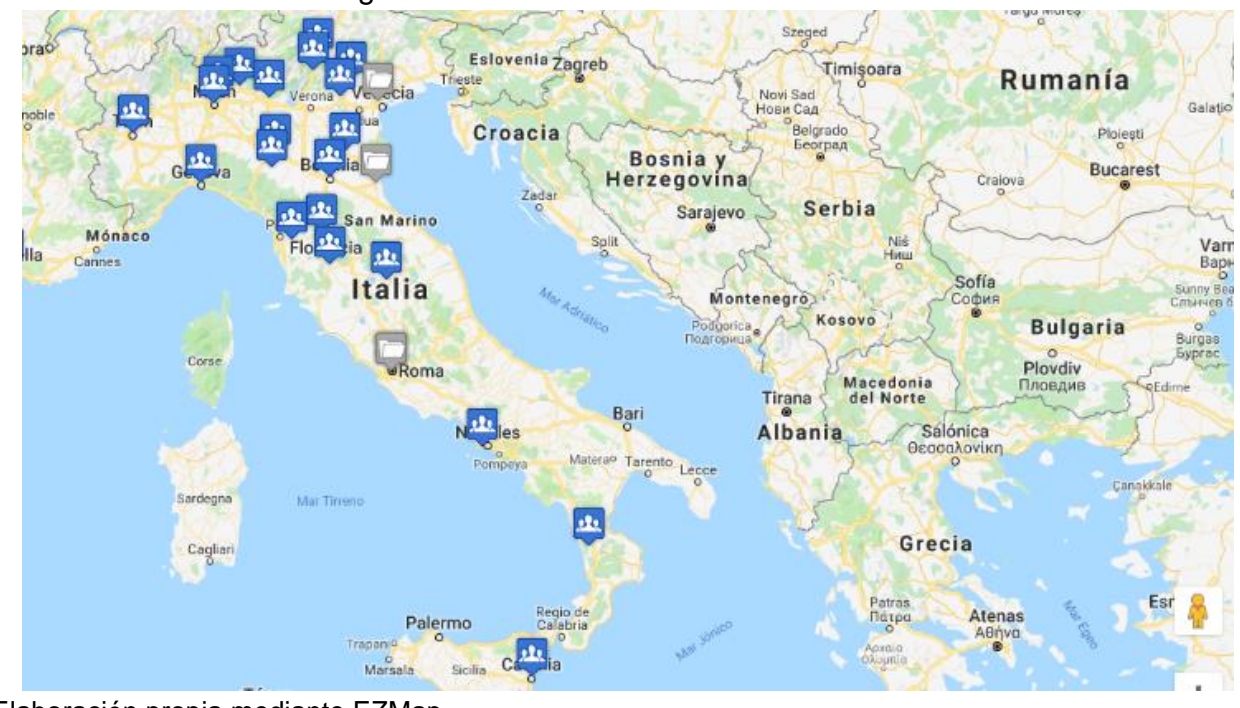

Fuente: Elaboración propia mediante EZMap

Tabla 4. Nombre e información de contacto de los UC en Italia

\begin{tabular}{ll}
\hline Italia & \\
\hline Urban Center Montalto Uffugo & sitio web: $\underline{w w w . c o m u n e . m o n t a l t o u f f u g o . c s . i t}$ \\
\hline Officina UrbaNa - Napoli & sitio web: $\underline{w w w . c o m u n e . n a p o l i . i t}$ \\
\hline Urban Center - Bologna & sitio web: $\underline{w w w . u r b a n c e n t e r b o l o g n a . i t ~}$ \\
\hline èFerrara - Urban Center & sitio web: $\underline{w w w . l a b o r a t o r i o u r b a n o . c o m u n e . f e . i t}$ \\
\hline Parma Urban Center & sitio web: $\underline{w w w . p a r m a u r b a n c e n t e r . i t}$ \\
\hline Urban Center Langhirano e Lesignano de' Bagni & sitio web: $\underline{w w w . c o m u n e l a n g h i r a n o . i t / u r b a n c e n t e r}$ \\
\hline Casa della Città - Roma & info: $06-67106286$ \\
\hline Urban Center di Genova & sitio web: $\underline{w w w . u r b a n c e n t e r . c o m u n e . g e n o v a . i t}$ \\
\hline Urban Center di Bergamo & sitio web: $\underline{w w}$ w.comune.bergamo.it \\
\hline
\end{tabular}




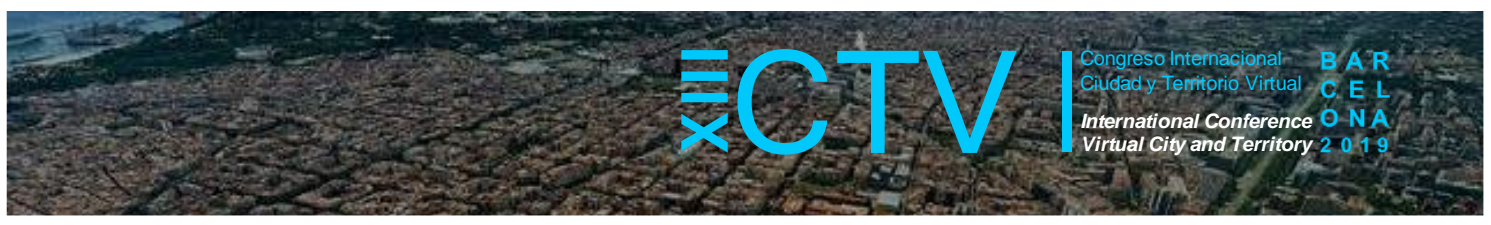

\begin{tabular}{|c|c|}
\hline Urban Center di Bergamo & sitio web: www.comune.bergamo.it \\
\hline Urban Center - Brescia & sitio web: http://www.comune.brescia.it/servizi/urbancenter \\
\hline Urban Center - Milano & sitio web: www.comune.milano.it/urbancenter \\
\hline Urban Center - Monza & e-mail: ufficiocomunicazione@comune.monza.it \\
\hline Urban Center Metropolitano - Torino & sitio web: $\underline{\text { www.urbancenter.to.it }}$ \\
\hline Casa della Città - Catania & sitio web: www.labpeat.dau.unict.it \\
\hline Urban Center Pontedera & sitio web: www.urbancenterpontedera.it \\
\hline Casa della Città di Siena & sitio web: www.comune.siena.it/La-Citta/Territorio \\
\hline Urban Center Metropolitano di Scandicci & sitio web: www.comune.scandicci.fi.it \\
\hline CasaCittà - Laboratorio urbano di Trento & e-mail: laboratorio urbano@comune.trento.it \\
\hline Urban Center Rovereto & sitio web: www.urbancenter.rovereto.tn.it \\
\hline Urban Center Perugia & e-mail: urbancenter@comune.perugia.it \\
\hline \multicolumn{2}{|l|}{ Urban Center Spoleto } \\
\hline Forum Center - Vicenza & sitio web: www.vicenzaforumcenter.it \\
\hline Urban Center Bassano & sitio web: www.urbancenterbassano.it \\
\hline \multicolumn{2}{|l|}{ Centros Urbanos Desactivados } \\
\hline Urban Center - Ravenna & $\begin{array}{l}\text { Sede expositiva: c/o Chiesa di S. Domenico - Via Cavour, } \\
1 \text { - } 48100 \text { Ravenna }\end{array}$ \\
\hline Urban Center Roma - XI Municipio & Via Niccolò Odero, 13 - 00154 Roma \\
\hline Urban Center di Venezia-Candiani 5 & Piazzale Candiani 5 - 30174 Mestre (VE) \\
\hline Urban Center Rovereto & sitio web: $\underline{w w w . c o m u n e . b e r g a m o . i t ~}$ \\
\hline
\end{tabular}

Estos centros en su gran mayoría, no suelen ser UC en su concepción sino centros culturales, debido a que el proceso de registro no tiene los filtros suficientes para que sólo UC se registren en la plataforma, pero nos sirve de referencia para entender que las ciudades han comprendido el papel fundamental de la participación ciudadana en los asuntos de ciudad y tratan de adaptar los espacios existentes en lugares donde se genera dicha participación. Por otro lado, encontramos los centros de innovación urbana, que van orientado al uso de las tecnologías para entender y mejorar la ciudad y la calidad de vida de sus habitantes, en la Tabla 5 podemos ver algunos ejemplos de estos en España y EE.UU.

Tabla 5. Centros de Innovación Urbana

\begin{tabular}{|c|c|}
\hline \multicolumn{2}{|l|}{ España } \\
\hline MediaLab Prado, Madrid & $\begin{array}{l}\text { sitio web: https://www.medialab-prado.es/medialab\#block- } \\
\text { medialab-theme-content }\end{array}$ \\
\hline Social Urban Lab Sant Feliu, Barcelona & sitio web: $\underline{w w w . s a n t f e l i u . c a t / i n n o v a}$ \\
\hline $\begin{array}{l}\text { Centro de Innovación Urbana Ca I'Alier -22@ BIT } \\
\text { Habitat, Barcelona }\end{array}$ & $\begin{array}{l}\text { sitio web: http://bithabitat.barcelona/en/ca-lalier/centre- } \\
\text { dinnovacio-urbana }\end{array}$ \\
\hline \multicolumn{2}{|l|}{ EE.UU. } \\
\hline MIT Senseable City Lab, Boston & sitio web: http://senseable.mit.edu/ \\
\hline
\end{tabular}

Los $\mathrm{CIU}^{8}$ suelen ser espacios que ponen a la disposición de los colectivos (ciudadanos, administración, empresas, universidades y centros de investigación) ${ }^{9}$ recursos tecnológicos para crear soluciones compartidas. Las actividades se gestionan en 4 aspectos fundamentales: proyectos de innovación, redes de innovación, estrategias de ciudad y espacios de colaboración. En este marco se plantea una fusión entre lo virtual y lo físico.

\footnotetext{
${ }^{8}$ Centros de Innovación Urbana

${ }^{9}$ Se trabaja la innovación con la implicación activa de actores de las denominadas "cuatro hélices": sector público, sector privado, sociedad civil y sector académico/investigador.
} 


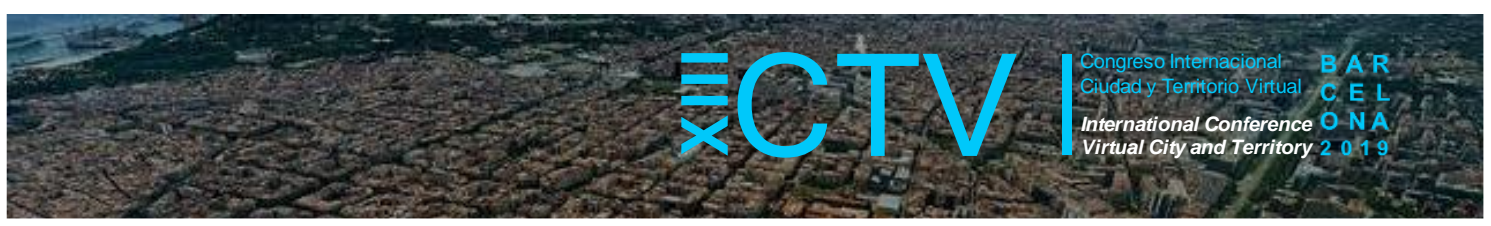

\section{Casos de Estudio}

\subsection{Urban Center Bolonia}

EL UC de Bolonia es un laboratorio abierto donde se difunden los principales proyectos y políticas estratégicas de Bolonia y su territorio metropolitano, donde los ciudadanos, los organismos públicos, las asociaciones y representantes del sector económico y social se reúnen y discuten las posibles transformaciones urbanas y territoriales y sus desafíos ambientales, sociales y tecnológicos (Bologna Welcome, 2019). Su objetivo principal es implicar al público en general en la transformación material e inmaterial de la ciudad.

Este centro es la sede de la fundación para la innovación urbana ${ }^{10}$ cuyas acciones se dirigen a cuatro ejes fundamentales que guían las políticas urbanas de Bolonia: Ciudad sostenible Nuevo bienestar urbano, Ciudad acogedora y Democracia urbana y digital, a través de tres vías principales: Urban center, donde se realizan actividades de información y promoción de la ciudad; Immaginazione civica, canales de participación y coproducción y Cartografare il presente, donde se analiza y documenta las transformaciones de la ciudad.(Fondazione innovazione urbana, 2019)

Este laboratorio urbano está distribuido en diferentes salas, la "Sala Atelier", "Il corner" y la "Sala Esposizioni", en las cuales se puede acceder a reuniones, seminarios, talleres y conferencias, que permitirá el acercamiento e involucramiento de las personas en los asuntos de ciudad. No sólo se puede participar a través del espacio físico del centro, sino también a través de medios digitales como su página web y apps. Tal es el caso de su proyecto BICIPLAN como plan de movilidad sostenible, en el cual todos los ciudadanos de Bolonia a través de sus dispositivos móviles pueden colaborar en el plan de movilidad mediante la aplicación Urban Decor, señalando aquellos puntos en la ciudad que requieren algún tipo de intervención.

\subsection{MIT Senseable City Lab}

Senseable City Laboratory es una iniciativa de investigación del Instituto de Tecnología de Massachusetts, el cual se enfoca en predecir los posibles cambios que puede sufrir la ciudad a través de su análisis desde un enfoque multidisciplinario: Diseño, planificación, planificadores, ingenieros, físicos, biólogos y científicos sociales. Las nuevas tecnologías nos abren un gran abanico de posibilidades para la gestión de la ciudad de manera colaborativo, sirviendo de interfaz de comunicación entre la ciudad y los ciudadanos (Ratti, 2012).

Este CIU se basa en el trabajo de investigadores desde diversos ámbitos del conocimiento para describir, diseñar y predecir comportamientos en la ciudad actual y en el futuro. Se toma como base datos existentes para predecir otros factores, por ejemplo, se realizó un modelo que se alimentó de datos de restaurantes chinos y tuvo como resultado la predicción de factores como la población diurna, la población nocturna, la presencia de la empresa y el monto del gasto.

\footnotetext{
10 Fondazione innovazione urbana es un centro de análisis, comunicación, elaboración y coproducción de transformaciones urbanas para enfrentar desafíos sociales, ambientales y tecnológicos (Fondazione innovazione urbana, 2019).
} 


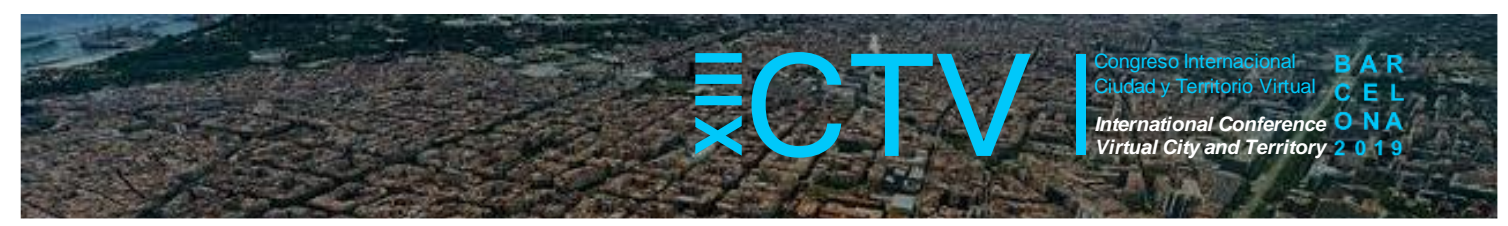

\subsection{Centro de Innovación Urbana Ca l'Alier-22@ BIT Habitat}

Este espacio de innovación es la sede de la fundación BIT Habitat ${ }^{11}$ cuyo objetivo es promover e impulsar la innovación urbana en Barcelona, en los aspectos sociales, económicos, urbanísticos y tecnológicos con la finalidad de responder a los nuevos retos que enfrenta la ciudad actual. Otra de las líneas de trabajo de esta institución es fomentar iniciativas focalizadas en la sostenibilidad de las ciudades y la calidad de vida de sus habitantes, mediante la cooperación entre ciudadanos, centros de investigación, universidades, empresas y la administración (BIT Habitat, s. f.).

El centro abrió sus puertas en septiembre del 2018 en la antigua fábrica Ca l'Alier rehabilitada con tales fines, situada en el cruce de las calles de Fluvià y Pere IV en el distrito Poblenou de Barcelona, en este se trabaja a partir de dos ejes o líneas estratégicas:

\section{- $\quad$ Taller de ciudades}

Espacio destinado a la difusión del pensamiento innovador, a través del intercambio de conocimiento, experiencias y estrategias entre las diferentes ciudades tanto nacionales como internacionales que posean los mismos retos de Barcelona (BIT Habitat, s. f.; Europa Press, 2018). "Se impulsarán actividades de encuentro entre ciudades, organizaciones e instituciones, tanto del ámbito ciudadano como de la investigación, el mundo empresarial y el sector públicopara compartir conocimientos y buenas prácticas. Se trabajará con grupos multidisciplinares y con la cuádruple hélice, alrededor de proyectos y retos urbanos definidos. Será también un espacio de divulgación del conocimiento en torno a los modelos urbanos eficientes, con utilización de datos y con el proyecto 22 @ como caso emblemático".(BIT Habitat, s. f.)

En el modelo de la Cuádruple Hélice las ciudades juegan un papel primordial en las estrategias de desarrollo y crecimiento, siendo la tecnología, la ciencia, la creatividad, la democracia y la inclusión social la base del mismo(Guillén, 2018).

\section{- I.Lab}

Es un espacio donde se experimenta a través de la colaboración de las cuatro hélices (ciudadanos, administraciones, empresas e instituciones formativas), esto permite la dinamización de las diferentes temáticas de interés dentro del ámbito de la innovación urbana (BIT Habitat, s. f.; Europa Press, 2018). "Se identificarán retos urbanos no resueltos aún por el mercado. Se abordará el desarrollo y testeo de nuevos productos y servicios que servirán para mejorar la vida de los ciudadanos. Esta tarea se llevará a cabo con el trabajo conjunto con ciudadanía, universidades y centros de investigación, empresas y administraciones".(BIT Habitat, s. f.)

Por otro lado, en el marco del proyecto BIT-Hábitat Urban Data Desk se ha desarrollado un proyecto piloto de plataforma web interactiva basada en GIS, para el estudio urbanístico del barrio y de la ciudad, permitiendo que el ciudadano pueda comprender, conocer, compartir y decidir sobre asuntos urbanos de su entorno (Ramos Sanz, Mayorga+Fontana Arq., Corolari. cat, Intelligent Moility, \& Col-lectiu El Tinglado, 2018).

\footnotetext{
${ }^{11}$ Barcelona Institute of Technology for the Habitat.
} 


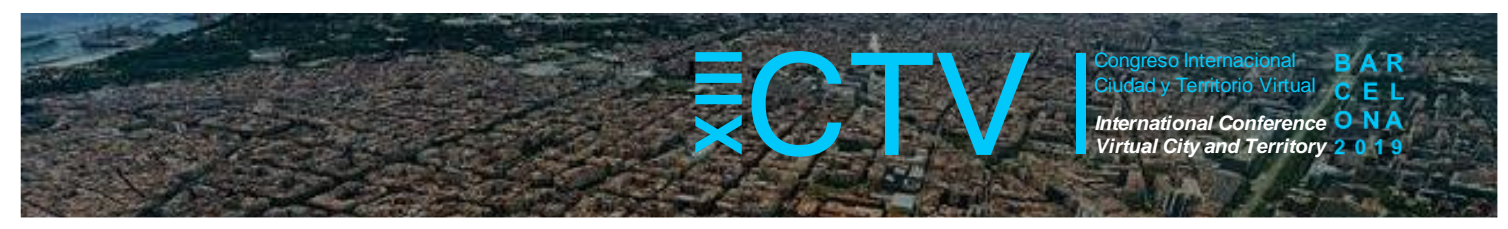

\section{UC, ClU y participación ciudadana}

"La participación es un fin es sí mismo. Es una actitud y una actuación cargada de otros valores sociales que la determinan también como valor humanizador. No solo participamos para construir un barrio mejor, sino que el hecho de participar alienta una puesta en común de experiencias, habilidades, actitudes preactivas, generación de cultura de equipo, comunicación ...., que en sí mismas ya gozan de un estatuto axiológico de envergadura."(Aranguren Gonzalo, 2005, pág.12)

Los UC y CIU, en el contexto de la participación, son la plataforma física de creación colectiva, partiendo del conocimiento como premisa y creando espacios abiertos para la confrontación, el debate, la crítica, la planificación y la gestión colaborativa de los asuntos urbanos, contribuyendo a las políticas de transformación del territorio y la ciudad (Tuttoggi.info, 2016).

\section{1 ¿Participación digital o presencial?}

Con el desarrollo y aumento de las TICs ${ }^{12}$, han proliferado nuevos mecanismos de diálogo y acceso a la información, lo cual ha propiciado una nueva perspectiva en las prácticas sociales y políticas. Esto ha conllevado una nueva forma de abordar la participación desde las tecnologías, propiciando una comunicación más fluida, efectiva y con costos más bajos, siendo punto de encuentro que posibilita el debate y la participación de diferentes colectivos (Manito, 2001).

En este ámbito, los recursos y plataformas de participación ciudadana han surgido como instrumentos fundamentales para promover la participación ciudadana en la co-creación de políticas públicas (Herranz, 2019), siempre que se empleen los métodos de trabajo en armonía con los ciudadanos.

La participación presencial, por su parte, nos permite una participación más directa, que facilita un involucramiento más duradero y efectivo de los actores del proceso, pero esta participación requiere de un esfuerzo mayor, para que el ciudadano esté motivado a realizarla, lo que amerita un conjunto de instrumentos y técnicas que permitirá la elección correcta de los actores y obtener los objetivos que se persiguen con dicho proceso participativo (Bustos, García, y Chueca, 2018) ${ }^{13}$.

Más allá de establecer cuál de estas dos modalidades es la más idónea para una participación más efectiva, debemos plantearnos cómo utilizar las herramientas actuales para lograr nuestro objetivo, a la vez que adaptamos dichos mecanismos de participación al contexto y a las necesidades del lugar donde serán aplicados, es decir, cada ciudad posee ciertas características que determinará si se puede implementar la participación presencial, la digital o tal vez, un modelo híbrido. (Ayuntamiento de Madrid, 2017; Coglobal, 2018; Rodríguez lbáñez, 2019).

\footnotetext{
12 Tecnologías de la Información y la Comunicación

13 Esta guía describe algunas pautas y recomendaciones generales para el diseño, ejecución y evaluación de procesos de participación y se detallan tanto una serie de técnicas y herramientas para el trabajo presencial como un conjunto de aplicaciones digitales que pueden resultar útiles para el desarrollo de dichos procesos.
} 


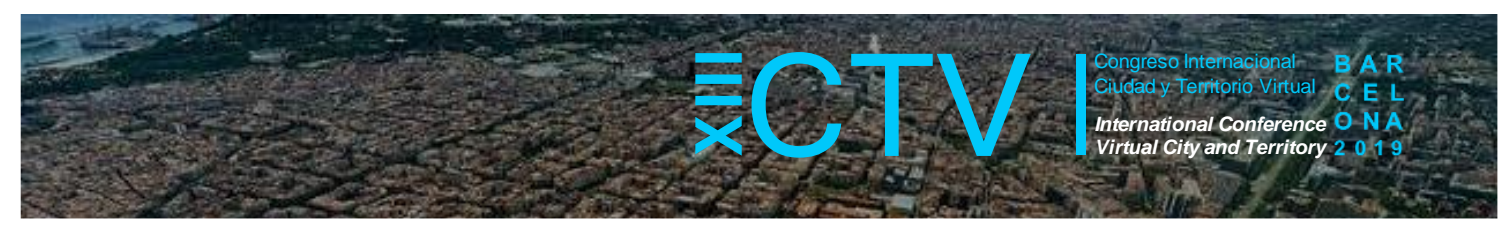

\section{Conclusión}

El acceso a la información es la base de todo proceso de participación, dicho esto, los UC promueven este conocimiento, a la vez que nos hace ser agentes fundamentales en la transformación y gestión de nuestra ciudad.

Al analizar el estado actual de los UC, se obtiene como resultado que la cobertura de éstos no es tan amplia como se desearía, estando localizados en Italia, Reino Unido, Austria, Alemania, Países Bajos, Francia, España, Canadá y EE. UU, además del hecho que muchos de estos no funcionan como UC sino como centros de exposición, por lo que no promueven activamente la participación de los ciudadanos. Por otro lado, este mecanismo de participación ciudadana, a pesar de los grandes esfuerzos que se vienen realizando desde unos años atrás no ha tenido una buena difusión en la mayoría de los países donde se han implementado, ni en países aledaños, al contrario de lo que pasa en Bolonia, donde se ha estado trabajando en la creación de una red de UC italianos, con ellos a la cabeza. A pesar de ello, existen dos casos interesantes analizados en este estudio: Boston, el cual está cambiando de centro a una red de centros, que permite el intercambio de experiencias, plantear problemas y soluciones comunes y satisfacer las necesidades específicas y generales de los ciudadanos y de las ciudades, algo parecido a lo planteado en Bolonia y Barcelona, donde se ha puesto en marcha un UC en el cual se fomenta la innovación urbana en los ámbitos social, urbanístico, económico y tecnológico. Estas buenas prácticas, aunque necesitan expandirse y renovarse, pueden servir como plataforma para la creación de una red de forma territorialmente descentralizada de puntos de referencia de participación ciudadana y de prácticas colectivas urbanas.

Entre los aspectos a considerar a la hora de entender los urban centers en la actualidad y sus posibles aplicaciones en las esferas de la participación son: sus limitantes como espacio físico (acceso, tiempo, movilidad, dinero), y el establecimiento de una relación entre estos y las nuevas tecnologías para obtener, una participación más inclusiva y cohesiva (plataformas y herramientas online).

A partir de lo analizado, es importante seguir tres líneas: 1)Transformar los ya existentes, para que incluyan a los ciudadanos no sólo como espectadores, sino como agentes activos en la conformación de la ciudad, a través de talleres, seminarios, debates, plataformas digitales, documentación, centros de exposición y mesas de trabajos, de la mano de las nuevas tecnologías de cara al usuario-ciudadano,2) Crear una red más amplia de UC, interconectadas unas con otras, para intercambiar experiencias y buenas prácticas, y 3) Crear herramientas 0 apoyarnos en las ya existentes, para una participación en tiempo real, más transparente y accesible.

Contribuciones de los autores: El primer, segundo y tercer autor han hecho aportaciones a la idea y diseño del estudio, a la recogida de datos, al análisis e interpretación de datos. El primer autor ha trabajado en la redacción del borrador del artículo y ha realizado la revisión crítica junto al segundo y tercer autor.

Conflicto de Intereses: Los autores declaran que no hay conflicto de intereses. 


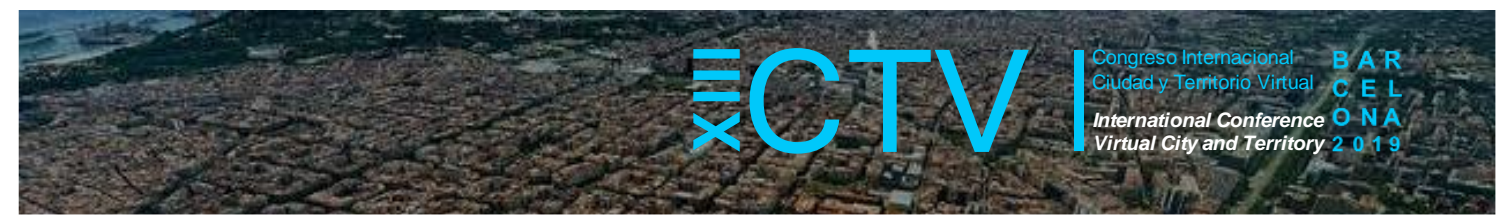

\section{Bibliografía}

Aranguren Gonzalo, L. (2005). La participación ciudadana: posibilidades y retos. Aposta. Revista de ciencias sociales, (22), 1-23. ISSN 1696-7348

Ayuntamiento de Madrid. (2017). Procesos de participación ciudadana realizados en la Ciudad de Madrid, junio 2015 - Julio 2017. Recuperado de https://www.oidp.net/docs/repo/doc216.pdf

BIT Habitat. (S. f.). Centro de Innovación Urbana Ca l'Alier BIT Habitat. Recuperado 17 de junio de 2019, de Bithabitat website: http://bithabitat.barcelona/es

Bologna Welcome. (2019). Urban center di Bologna. Recuperado 6 de septiembre de 2019, de Bologna Welcome website: https://www.bolognawelcome.com/home/scopri/luoghi/cultura-estoria/musei-e-gallerie-arte/urban-center-di-bologna/

Borja, J., \& Castells, M. (1997). Local y global. La gestión de las ciudades en le era de la información. Madrid, España: Santillana, S.A. Taurus.

Bustos, R., García, J., \& Chueca, D. (2018). Guía práctica para facilitar la participación ciudadana. Una selección de herramientas presenciales y digitales para el trabajo colectivo. Navarra, España: Departamento de Relaciones Ciudadanas e Institucionales.

Coglobal. (2018). Entre lo digital y lo presencial: nuevas formas de participación. Recuperado 12 de septiembre de 2019, de Coglobal website: http://www.coglobal.es/entre-lo-digital-y-lopresencial-nuevas-formas-de-participacion/

Europa Press. (2018). Ca l'Alier, de antigua fábrica a centro puntero de innovación urbano y tecnológico. La Vanguardia. Recuperado de https://www.lavanguardia.com/local/barcelona/20180607/444194433945/acaban-las-obras-deca-lalier-para-albergar-un-centro-de-cisco-y-de-innovacion-urbana.html\#linkcomments

Fernández González, M. (2015). La Smart City como imaginario socio-tecnológico. La construcción de la utopía urbana digital (Tesis doctoral). Universidad del País Vasco, Vitoria, España. DOI: https://doi.org/10.20868/ciur.2016.109.3498

Fondazione innovazione urbana. (2019). Fundación para la innovación urbana. Recuperado 6 de septiembre de 2019, de http://www.fondazioneinnovazioneurbana.it/chisiamo

Guillén, D. (2018). Cuádruple hélice en la gestión territorial. Revista Cataluña Económica, (540), 57-59. ISSN: 1135-819X.

Herranz, C. (2019). Comunidades y redes: la base de la participación digital efectiva. Recuperado 12 de septiembre de 2019, de Analítica Pública website: https://analiticapublica.es/comunidades-y-redes-la-base-de-la-participacion-digital-efectiva/

Manito, F. (2001). Participación ciudadana y Internet. Recuperado 12 de septiembre de 2019, de Barcelona.cat website: http://www.bcn.cat/publicacions/bmm/54/cs qc06.htm 


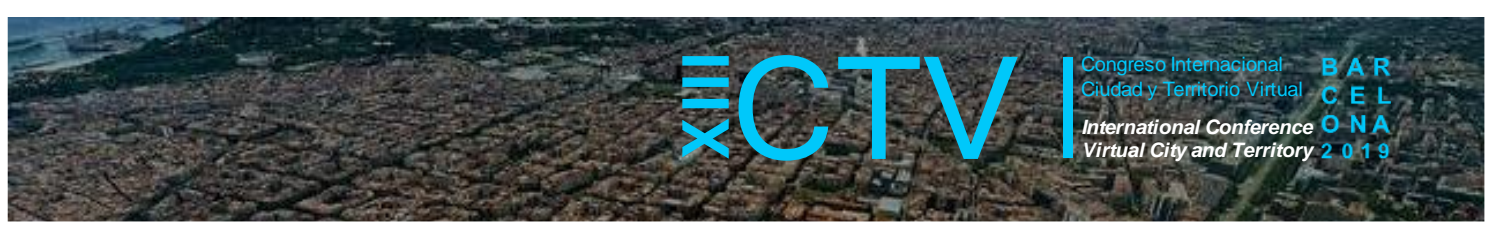

Observatorio de investigación del centro urbano. (S. f.). Urban Center. Recuperado 6 de mayo de 2019, de Urban Center website: http://www.urban-center.org/en/

Ramos Sanz, M., Mayorga+Fontana Arq., Corolari. cat, Intelligent Mobility, \& Col-lectiu El Tinglado. (2018). Informe d'assistència a la implementació de l'Urban Data Desk (UDD) de BITHàbitat. Ajuntament de Barcelona, Barcelona.

Ratti, C. (2012). Senseable City Lab. Recuperado 5 de septiembre de 2019, de MIT urban planning website: https://dusp.mit.edu/uis/project/senseable-city-lab

Rodríguez Ibáñez, M. (2019). Participación ciudadana: de las plataformas presenciales y virtuales. Trabajo presentado en: Sharing society international conference, Madrid, España. Recuperado de https://www.academia.edu/36849541/Participación Ciudadana de las Plataformas Presencial es y Virtuales

Sierra Caballero, F., \& Del Valle, C. (2007). Nuevas tecnologías de la información y participación ciudadana. Redes.com: revista de estudios para el desarrollo social de la Comunicación, (4), 17-19. DOI: https://doi.org/10.15213

Tuttoggi.info. (2016, febrero 18). Centro urbano también en Spoleto, para diseñar la ciudad del futuro. Tuttoggi.info. Recuperado de https://tuttoggi.info/urban-center-anche-a-spoleto-perprogettare-la-citta-del-futuro/320408/ 